\title{
Improvements in Mindfulness Facets Mediate the Alleviation of Burnout Dimensions
}

\author{
Sanna M. Kinnunen ${ }^{1}\left(\mathbb{D} \cdot\right.$ Anne Puolakanaho $^{1} \cdot$ Asko Tolvanen $^{1} \cdot$ Anne Mäkikangas $^{2} \cdot$ Raimo Lappalainen $^{1}$
}

Published online: 27 August 2020

(C) The Author(s) 2020

\begin{abstract}
Objectives While interventions using mindfulness have been effective in treating burnout, the mechanisms of change need more research. This study investigated which of five mindfulness facets (observing, describing, acting with awareness, non-judging, and non-reacting) mediated the intervention effects on three burnout dimensions (exhaustion, cynicism, and reduced professional efficacy) during an 8-week mindfulness-, acceptance-, and value-based (MAV) intervention and a 10-month follow-up.

Methods The participants were a heterogeneous sample of employees suffering from burnout $(n=202,80 \%$ women, mean age $=47.5$ years). Latent change score modeling was conducted for each combination of the mindfulness facets and the burnout dimensions. Confidence intervals were calculated for the coefficients in the models.

Results The modeling results showed that mindfulness improvement during the intervention mediated burnout alleviation during both the intervention and the 10-month follow-up. A large spread of mindfulness facets mediated changes in all the burnout dimensions during the intervention (all for cynicism, all except describing for exhaustion, and all except observing for reduced professional efficacy). The improvement in non-judging skills mediated the reductions in all burnout dimensions during the follow-up. For exhaustion, it was the only significant mediator during the follow-up, whereas for cynicism and reduced professional efficacy, describing and observing were additional mediators.

Conclusions Improving mindfulness facets using a MAV intervention had significant long-term effects on burnout in this study. Non-judging is possibly the most important mindfulness facet to improve in burnout interventions, given that it mediated the changes in all burnout dimensions during both the intervention and 10-month follow-up.
\end{abstract}

Keywords Mindfulness $\cdot$ Burnout $\cdot$ Mediation $\cdot$ Intervention $\cdot$ Acceptance and commitment therapy

Burnout has been associated with increased mental and physical health problems, and negative organizational outcomes, such as increased employee turnover, sickness absences, and impaired job performance (Ahola et al. 2017; Morse et al. 2012). Burnout is a persistent, job-related state of ill-being that is characterized by exhaustion, cynicism, and reduced

Electronic supplementary material The online version of this article (https://doi.org/10.1007/s12671-020-01490-8) contains supplementary material, which is available to authorized users.

Sanna M. Kinnunen

sanna.kinnunen@mielenhuolto.fi

1 Faculty of Education and Psychology, University of Jyväskylä, PO Box 35, FI-40014 Jyväskylä, Finland

2 Faculty of Social Sciences (Psychology), University of Tampere, 33014 Tampere, Finland professional efficacy (Leiter et al. 2014; Näätänen et al. 2003). Exhaustion refers to feelings of physical and emotional fatigue, cynicism to distancing oneself from work and questioning the meaningfulness of one's job, and reduced professional efficacy to perceiving one's capabilities as inadequate for satisfactory job performance. Burnout has been reluctant to change without intervention (Mäkikangas and Kinnunen 2016). Therefore, it is essential to develop effective treatments to mitigate its adverse effects. It is also important to understand by which processes these interventions work. By emphasizing the intervention processes that are the most likely to alleviate burnout, intervention effectiveness could likely be improved, and resources used cost-effectively. Process-based intervention research is a way to increase process knowledge since it focuses on studying theory-based and empirically supported processes responsible for positive intervention outcomes (Hofmann and Hayes 2019). Process-based research is interested in whether the manipulation of a certain process 
is efficacious in reducing the targeted symptoms and whether the used intervention can manipulate the process intendedly (Hayes and Hofmann 2017; Hofmann and Hayes 2019). Hofmann and Hayes (2019) suggested that different procedures could influence the same process. Hence, diverse interventions could lead to similar outcomes.

In interventions using mindfulness, it is a central, theoretically expected mechanism of change (Crane et al. 2017; Hayes et al. 2012). Fletcher and Hayes (2005) offered a functional definition of mindfulness that includes elements from several conceptualizations of mindfulness and integrates diverse mindfulness processes. The functional mindfulness definition comprises processes of staying in the present moment, taking an open and non-judgmental stance towards one's experiences, detaching from the guidance of one's thoughts and feelings, and transcending the conceptual description of oneself. In empirical research, a measure that is well-suited for studying different mindfulness processes is the Five Facet Mindfulness Questionnaire (FFMQ, Baer et al. 2006). It evaluates five mindfulness facets, namely observing, describing, acting with awareness, non-judging, and non-reacting. Observing refers to noticing inner and outer stimuli, such as sensations, thoughts, and feelings; describing is the ability to describe observed stimuli with words; acting with awareness refers to being aware of one's situation and acting with conscious intention rather than reacting automatically; nonjudging means refraining from evaluating one's thoughts, feelings, and sensations; and non-reactivity is the ability to let thoughts and feelings come and go without becoming attached or impulsively reacting to them. Concerning the functional definition of mindfulness by Fletcher and Hayes (2005), observing and describing depict the skills to be in contact with the present moment, while non-judging reflects the ability to take an accepting stance towards the experiences at the moment. Non-reacting and acting with awareness depict the skills to defuse from the behavioral guidance of thoughts and feelings and instead act according to one's personal goals.

Recently, interventions using mindfulness have shown promise in burnout treatment (Luken and Sammons 2016; Lloyd et al. 2013). Mindfulness has also been the mediator of burnout change in these interventions (Roeser et al. 2013). However, recent cross-sectional studies have indicated that certain mindfulness processes could be more relevant to positive intervention outcomes than others. Yang et al. (2017) found that, while all five mindfulness facets of the FFMQ had a negative association with exhaustion and disengagement (comparable with cynicism), the strongest association was found for acting with awareness. Kriakous et al. (2019) also observed that higher levels of acting with awareness were associated to lower levels of emotional exhaustion and depersonalization. Taylor and Millear (2016) separated between the burnout dimensions and found that non-judging and non-reacting were associated with emotional exhaustion, acting with awareness, and non-judging with cynicism, and observing with reduced professional efficacy. For each facet, higher mindfulness was associated with lower burnout. These results indicate that, instead of studying mindfulness as a unified mediator, researchers should study its processes as separate mechanisms of change in the alleviation of burnout.

Although the research on the associations between mindfulness facets and burnout is mostly cross-sectional, there is evidence of the differential role of mindfulness facets in the changes of other well-being indicators during the intervention. Either non-judging or acting with awareness predicted positive intervention outcomes in terms of stress, anxiety, work-related rumination, and fatigue (Bergman et al. 2016; Querstret et al. 2016, 2018). However, with some well-being indicators, simultaneous improvements in observation-related (observing or describing) and reaction-related (non-judging or non-reacting) facets were needed to induce positive change. This need was noticed for psychological distress, psychological symptoms, and depression (Heeren et al. 2015; Querstret et al. 2018; Waters et al. 2018). More process research is needed to understand better which combination of mindfulness facets would show the most beneficial results in burnout treatment. Furthermore, the findings of Taylor and Millear (2016) suggest that different mindfulness facets could be important for the alleviation of separate burnout dimensions, indicating that it is warranted to study the associations between mindfulness processes and burnout separately for each dimension.

The current study aimed to understand better how interventions using mindfulness achieve their positive effects. This study explored whether the five facets of mindfulness, according to the FFMQ (observing, describing, acting with awareness, non-judging, and non-reacting), were mediators of burnout change during an 8-week intervention and a 10-month follow-up. Furthermore, this study investigated the differences between the burnout dimensions (exhaustion, cynicism, and reduced professional efficacy) regarding how the mindfulness facets mediated their changes. Mindfulness facets were expected to mediate the intervention effects on the burnout dimensions, but no specific hypotheses were made about the associations between the separate mindfulness facets and burnout dimensions.

\section{Method}

\section{Participants}

The current study was conducted as a part of a randomized controlled trial (RCT) titled The Effectiveness of Mindfulness Practices in the Recovery of Burnout, funded by the Finnish Social Insurance Institution and registered under ClinicalTrials. gov (NCT number: NCT01920230). The research protocol was approved by the Research Ethics Committee of the Central 
Finland Health Care District. As part of the ethical evaluation, a report on the data processing and storage was approved. Data were stored anonymously in the university data storage. The results of the RCT are presented in Puolakanaho et al. (2020). Participants were recruited using web page announcements and newspaper advertisements and via occupational health care units. Enrollment took place via a web questionnaire and was open to anyone interested in participating in the study. The selection was based on information provided by the applicants via the enrollment questionnaires and subsequent interviews. The inclusion criteria were the age of 25-60 years, ongoing work, daily available Internet connection, and high burnout according to the cutoff score of the Bergen Burnout Indicator (BBI). The BBI cutoff was the 75th percentile (39-47 points) of the age group, according to the manual by Näätänen et al. (2003). Candidates were excluded if they had serious psychological or somatic disorders, were in regular psychotherapy, or were susceptible to major pharmaceutically induced mood swings. The participants gave their informed consent and received the intervention free of charge. They were emailed web questionnaires before the intervention (pre), after the intervention (post), and 10 months after the post-measurement (fup10). The pre-measurement was completed within 2 weeks before the intervention started. Reminders were sent via email and telephone.

The participants were paired based on sex, age, and education. Then, either participant in each pair was assigned to an intervention group receiving an intervention in addition to treatment-as-usual (TAU; $n=133 ; 12$ separate groups), and the other was assigned to a control group receiving only TAU $(n=133)$. Most of the participants $(82 \% ; n=109$ for both groups) were blindly randomized to the groups. The remaining participants ( $18 \%, n=24$ for both groups) were matched without randomization using the same criteria (sex, age, and education). This was done to allow for a sophisticated statistical analysis of the associations between the mindfulness facets and the burnout dimensions, given that a larger sample size was required to fit the criteria for structural equation modeling (Wolf et al. 2013).

The same inclusion criteria and matching procedure were applied for both the randomized and non-randomized participants to mitigate the risk of confounding variables affecting the results of the study. A pilot study with 24 intervention participants was conducted before the RCT, and a matching procedure was completed separately to obtain corresponding control participants for the pilots. These controls were collected from the participants who initially enrolled in the study and fulfilled the inclusion criteria but were not able to participate in the face-to-face meetings and were thus not randomized. The non-randomized intervention participants underwent the same intervention procedure as the randomized intervention participants, and the non-randomized control participants underwent the same procedure as the randomized control participants.
No significant differences were found between the randomized and non-randomized controls in terms of sex, age, education, and the main study variables (five mindfulness facets and three burnout dimensions) at the enrollment, pre-, post-, or fup10 measurements, as shown by an independent samples $t$ test. The same was observed for differences between the randomized and non-randomized intervention participants, except that the latter experienced less exhaustion at the enrollment and had lower reduced professional efficacy at the premeasurement than the former. The exact results of all the $t$ tests in the methods, with the means and standard deviations for the compared groups, are reported in the supplementary material (Appendix A).

Sample Attrition Initially, there were 266 participants (133 each in the intervention and control groups). Before completing the post-measurement questionnaire, 63 participants withdrew from the study. A total of $41 \%$ of these dropouts $(n=26)$ came from the intervention group, and $59 \%(n=37)$ were from the control group. In addition, one participant from the intervention group was excluded from the analyses because their BBI score dropped significantly between enrollment and premeasurement (randomization was completed in the enrollment phase, when the BBI score matched the inclusion criteria). An independent samples $t$ test revealed no significant differences between those who dropped out and those who remained in terms of sex, age, education, or initial level of reduced professional efficacy. However, those who dropped out experienced more exhaustion and cynicism at the enrollment phase than those who stayed.

The sample that was used in the analyses consisted of 106 intervention group participants and 96 control group participants, yielding a total sample of 202 participants. All of them completed both the pre- and post-measurements. By the 10th month after the post-measurement, 23 participants had dropped out of the study. There were no significant differences between those who remained in the study and those who dropped out in any of the demographic or main study variables, according to an independent samples $t$ test. A diagram of the participant flow is presented in the supplementary material (Appendix A).

The participants of the final sample were all Caucasian, and $80 \%$ of them were women. The mean age of the participants was 47.5 years ( $\mathrm{SD}=8.05$, a range of $27-60$ years), and $67 \%$ of them had a polytechnic or university degree. Of the participants, 30\% had vocational education, and 3\% had participated in other forms of education (e.g., short employment courses). They worked approximately $40 \mathrm{~h}$ per week (SD = 9.55). Of the participants, $75 \%$ were married or cohabiting, $13 \%$ were divorced or widowed, and $11 \%$ were single. Ten percent evaluated their economic situation as very good, $57 \%$ 
rated it as rather good, 29\% described it as rather tight, and 4\% considered it very tight.

\section{Procedures}

Intervention The 8-week intervention combined weekly group meetings with the use of a web program. The main structure and most homework assignments were based on the mindfulness intervention by Williams and Penman (2011). Theoretically, the intervention was founded on the principles of ACT (Hayes et al. 2012). In line with this, the informatory content was formed based on ACT. Thus, the present intervention combined elements of traditional mindfulness-based intervention and ACT to increase the mindfulness and acceptance skills and promote the valuebased actions of the participants. Therefore, the program was described as mindfulness-, acceptance-, and value-based (MAV) intervention. The intervention included weekly themes related to identifying the factors contributing to one's burnout, changing routines to support one's well-being, and learning new ways to relate to one's situation. The participants practiced self-compassion and a non-judging stance towards their experiences. They also practiced letting go of attempts to control their thoughts and feelings. The participants were instructed to perform formal mindfulness-acceptance practices (e.g., 10-15 min body scan or loving-kindness meditation) twice a day for 6 days a week. The formal practices also included a short breathing space that was to be done once a day. In addition, the participants were instructed to mindfully carry out informal activities, such as daily tasks, and change their routines. They were further advised to perform valuebased actions in their daily lives. Homework assignments and voluntary material were available through the web program. The intervention was standardized and delivered by two psychologists with experience and training related to the practices used. Adherence to the protocol was relatively high in terms of participation in group meetings and completion of homework. A detailed description of the contents and adherence is presented in the supplementary material (Appendixes $\mathrm{B}$ and $\mathrm{C}$ ).

TAU In the research project to which this study belongs, the purpose was to investigate whether adding a MAV intervention to TAU would have superior effects compared with those of only TAU in burnout alleviation. Therefore, both the intervention and control group participants could use TAU services. Furthermore, for ethical and practical reasons, the researchers did not advise the intervention participants not to use the services. The control group participants were especially encouraged to use the available services, but they were not directed to any service by the research group. Furthermore, the control group did not receive any intervention from the research group, but they were promised access to the web program after the 12-month study period was over. In Finland, several approaches are used to alleviate burnout symptoms, such as therapeutic conversations in employee health care, rehabilitation, sick leaves, medication, support from employers, and changes in job conditions. Sixty-three percent of the intervention and $62 \%$ of the control participants utilized at least one support form during the intervention. During the follow-up, $70 \%$ of the intervention and $80 \%$ of the control participants used TAU services. Details about the used services are presented in the supplementary material (Appendix C).

\section{Measures}

Burnout was measured using the 15-item BBI (Näätänen et al. 2003). The BBI-15 has a subscale for each burnout dimension: exhaustion (five items, e.g., "I am snowed under with work"), cynicism (five items, e.g., "I feel dispirited at my work and I think of leaving my job"), and reduced professional efficacy (five items, e.g., "I frequently question the value of my work"). The 6-point response scale for this questionnaire ranges from 1 (completely disagree) to 6 (completely agree). This measure was chosen since it contains cutoff scores for Finnish samples and could thus be used to include only workers with the highest amount of burnout symptoms. The reliability and validity of this measure have been found to be relatively good by previous studies (Salmela-Aro et al. 2011; Näätänen et al. 2003).

The mindfulness facets were measured using the FFMQ (Baer et al. 2006). This questionnaire consists of 39 items measuring five mindfulness facets: observing (eight items, e.g., "When I'm walking, I deliberately notice the sensations of my body moving"), describing (eight items, e.g., "I'm good at finding words to describe my feelings"), acting with awareness (eight items, e.g., "When I do things, my mind wanders off and I'm easily distracted", reverse-scored), non-judging (eight items, e.g., "I criticize myself for having irrational or inappropriate emotions", reverse-scored), and non-reacting (seven items, e.g., "I perceive my feelings and emotions without having to react to them"). The 5-point response scale for this questionnaire ranges from 1 (never or very rarely true) to 5 (very often or always true). This measure was chosen since it has separate subscales for different facets. In previous studies, the reliability and validity of this measure have been relatively good (Baer et al. 2006, 2008). Cronbach's alphas from pre- to fup 10 measurements for burnout and mindfulness are presented in Table 1.

\section{Data Analyses}

Descriptive statistics (means, standard deviations, correlations, and reliability calculations) were computed with SPSS Statistics 24, and other analyses were completed with Mplus 8 


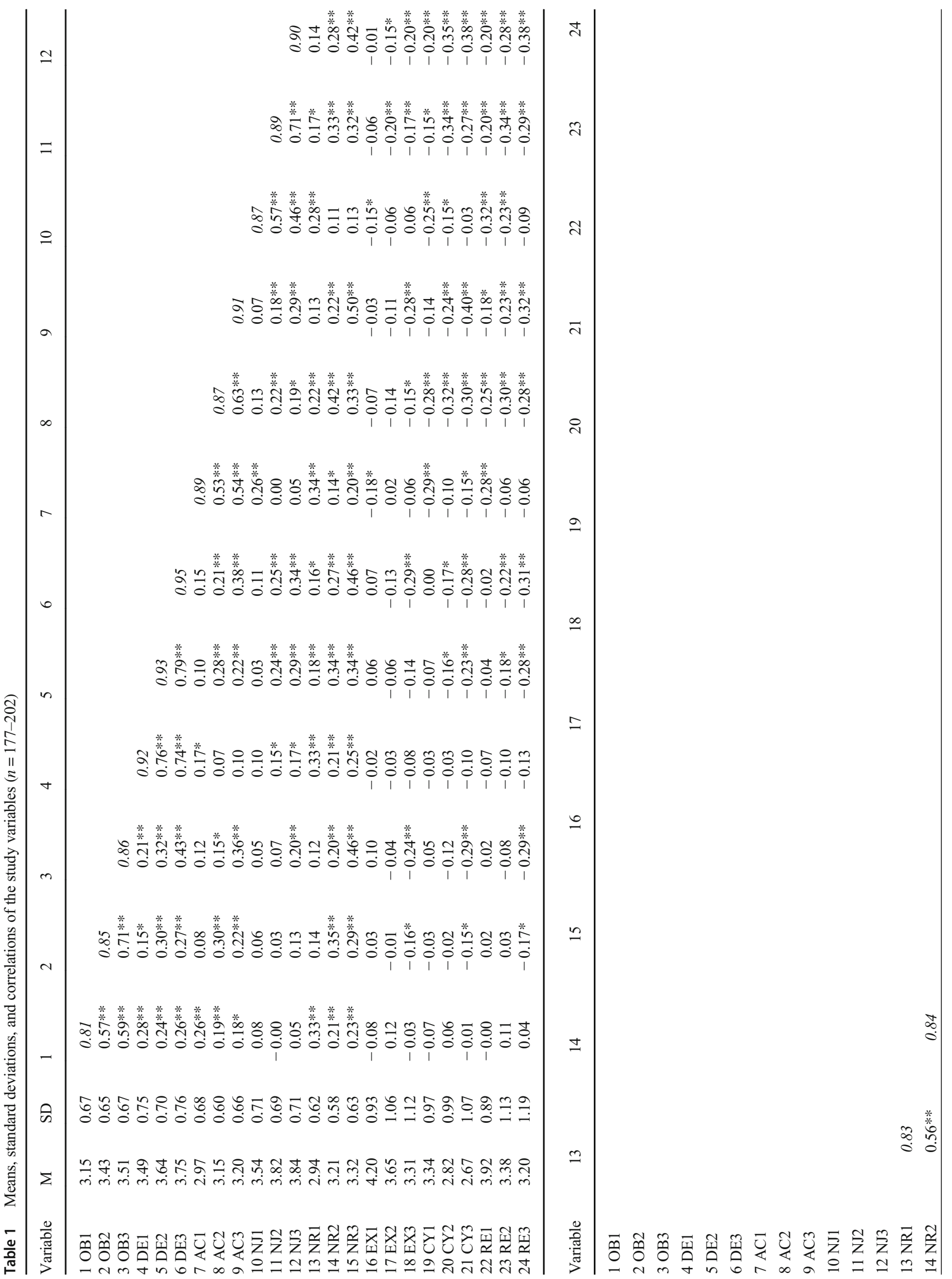




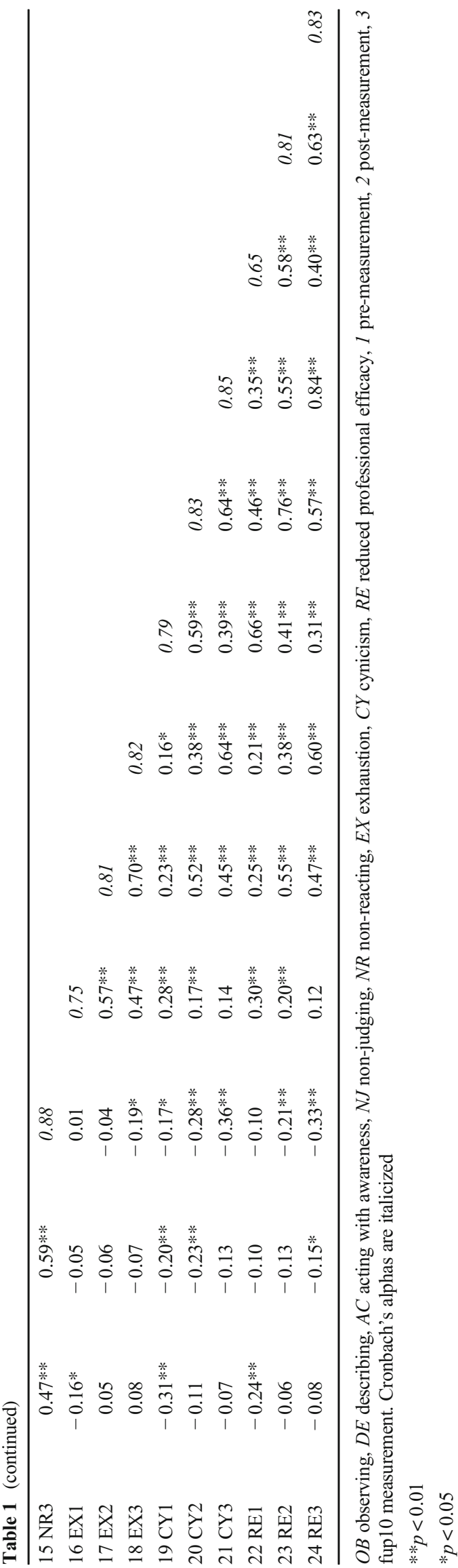

(Muthén and Muthén 2017). Latent change score modeling (McArdle and Hamagami 2001) with a measurement model was conducted for each combination of the mindfulness facets and burnout dimensions, yielding 15 independent models. The used model was the same for each combination and is presented in Fig. 1. Reliability statistics (Cronbach's alphas) were calculated for the mindfulness and burnout scales, and these results showed good or excellent values $(\alpha=0.81-0.95)$ for all mindfulness facets at all time points and for all burnout dimensions at the post- and fup 10 measurements. At the premeasurement, the alpha values were adequate for exhaustion ( $\alpha=0.75)$ and cynicism $(\alpha=0.79)$ but questionable for reduced professional efficacy $(\alpha=0.65)$. The measurement model was used to further eliminate the effect of error variance on the study constructs and to ensure the reliability of the constructs in the final model. To improve the variable-tosample size ratio, construct-specific parcels were created according to recommendations from Little et al. (2002). Previously identified structures of the five mindfulness facets (Baer et al. 2006) and the three burnout dimensions (Näätänen et al. 2003) were utilized as bases for parcel creation. Confirmatory factor analyses (CFAs) were conducted at pre-, post-, and fup10 measurements for each construct to validate the use of these existing structural definitions for the mindfulness facets and burnout dimension. Since the CFA models fitted the data reasonably well and the factor loadings of the individual items were approximately the same size for the given factors, the parcels were created by combining the items in the order they are presented in the original questionnaire. This way, the individual items of each mindfulness facet were divided into three parcels, as were the items of each burnout dimension. In the parcels, an individual level that was stable over time and was unassociated with any other part of the model was detected. This was considered by adding a level correction to the model for the parcels. The scalar equivalence (e.g., equal factor loadings and equal intercepts of observed variables) was expected to hold across time.

Latent change score modeling combines features from cross-lagged regression modeling and latent growth curves (McArdle 2009; McArdle and Hamagami 2001). In latent change score modeling, variable $Y$ is described at a time $t$, and $\Delta Y_{t}$ is defined as the change in $Y$ from $t-1$ to $t$ (McArdle 2009). In the current study, the change scores were calculated for the factors instead of observed variables; hence, $Y$ here referred to a factor. The coefficients relating to $Y_{t}$ and $Y_{t-1}$ were constrained to 1 , and there were no error terms in the equation for $Y_{t} . Y_{t}$ was a direct sum of $Y_{t-1}$ and $\Delta Y_{t}$. This way, $\Delta Y_{t}$ could be used as a latent variable that directly indicated the amount of change in the target variable between given time points. Latent change scores were created for the changes from pre- to post-measurement and from post- to fup 10 measurement in the mindfulness facets and burnout dimensions (marked D in the figure). Modeling was conducted, adjusting 


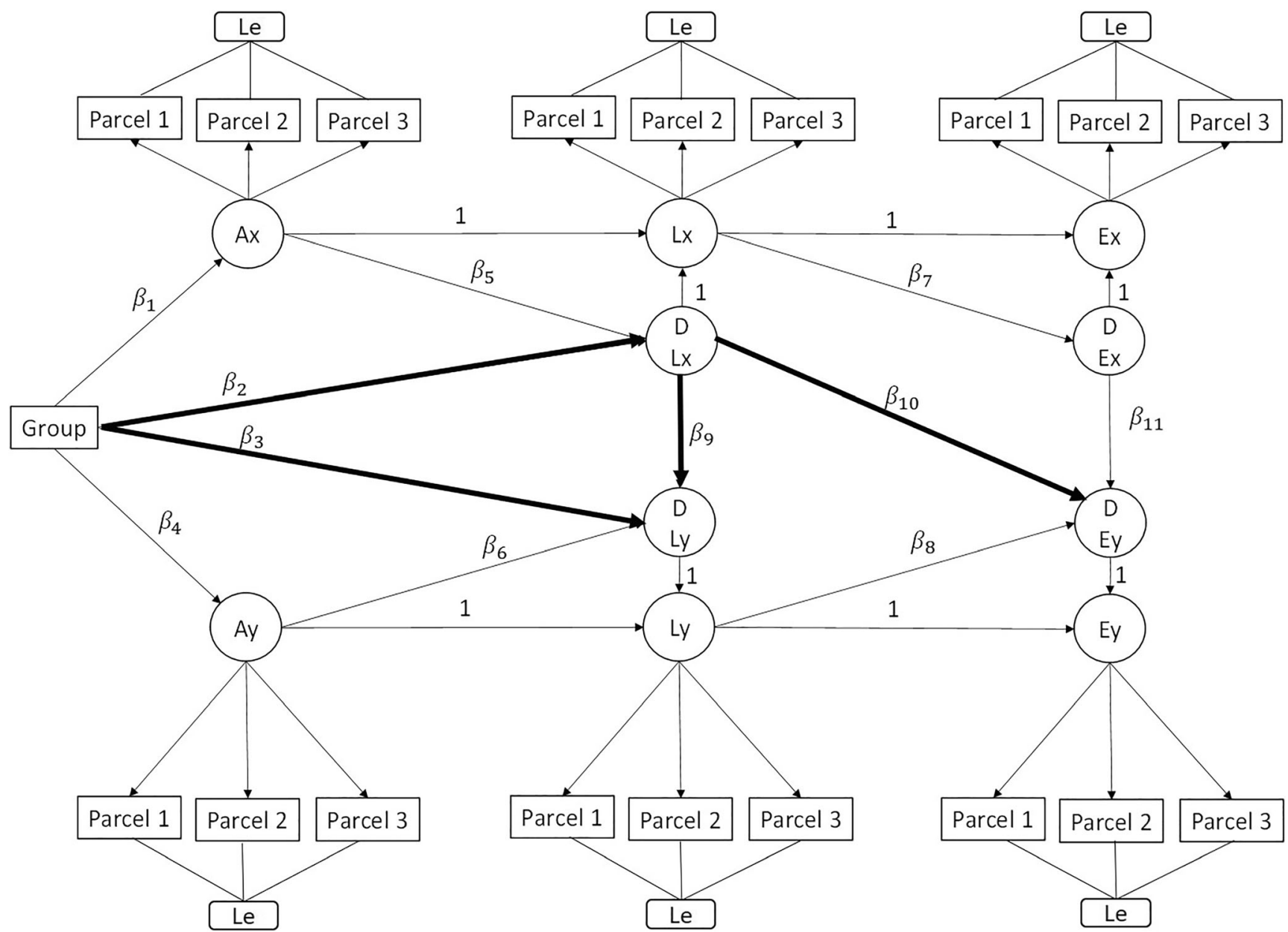

Fig. 1 Latent change score model of mindfulness ( $x$ ) and burnout (y). The same model was used separately for each combination of mindfulness facets and burnout dimensions. The essential pathways are bolded. $\mathrm{A}=$ pre, $\mathrm{L}=$ post, $\mathrm{E}=$ fup $10, \mathrm{D}=$ latent difference score, $\mathrm{Le}=$ individual level.

the effect of non-normality with a robust full information maximum likelihood estimator. A few outliers represented real observations of the participants with the different intervention effects; thus, they were included in the models. The possible effects of these outlier observations on the study results were evaluated by comparing the results after the exclusion of problematic observations. These examinations showed no significant changes to the results. Standardized model results were reported, from which the magnitudes of the effects were directly observable without further effect size calculations.

The fit of the models was evaluated using the chi-square test $\left(\chi^{2}\right)$, comparative fit index $(\mathrm{CFI})$, Tucker-Lewis index (TLI), standardized root mean square residual (SRMR), and root mean square error of approximation (RMSEA). As recommended by $\mathrm{Hu}$ and Bentler (1999), a good model fit was assumed when the CFI and TLI were close to 0.95, the SRMR was close to 0.08 , and the RMSEA was close to 0.06 .

As shown in Fig. 1 (relevant pathways are bolded), $\beta_{2}, \beta_{9}$, and $\beta_{10}$ were the most relevant coefficients to the research
Previously identified structures of the five mindfulness facets (Baer et al. 2006) and three burnout dimensions (Näätänen et al. 2003) were utilized as bases for parcel creation, and the items of both measures were divided into three parcels

questions about the mediation effects. $\beta_{2}$ showed whether the intervention affected the change from pre- to postmeasurement in each mindfulness facet. $\beta_{9}$ and $\beta_{10}$ showed whether the change in a mindfulness facet from pre- to postmeasurement predicted the change in a burnout dimension from pre- to post-measurement $\left(\beta_{9}\right)$ and from post- to fup 10 measurement $\left(\beta_{10}\right)$. Furthermore, the pathways $\beta_{2} \times \beta_{9}$ and $\beta_{2}$ $\times \beta_{10}$ showed whether the effects of the intervention on the burnout dimensions were mediated by the changes in the mindfulness facets (indirect effects). $\beta_{2} \times \beta_{9}$ showed how the change in the mindfulness facets during the intervention was connected to the change in the burnout dimensions during the intervention. Thus, mindfulness and burnout were measured at the same time points. In addition, $\beta_{2} \times \beta_{10}$ showed how the change in the mindfulness facets during the intervention was connected to the change in the burnout dimensions during the follow-up. Thus, mindfulness change was measured before burnout change. $\beta_{3}$ showed whether the intervention had an additional direct effect on the change from pre- to 
post-measurement in the burnout dimensions after the mediated indirect effect was considered. A 95\% confidence interval for the coefficients (estimate \pm 2 standard errors) was calculated to evaluate the differences between the burnout dimensions with regard to the significant predictors of change. Overlapping of the confidence intervals for the equivalent coefficients $\left(\beta_{2} \times \beta_{9}, \beta_{2} \times \beta_{10}, \beta_{2}, \beta_{9}\right.$, and $\left.\beta_{10}\right)$ of the mindfulness facets in the case of each burnout dimension indicated that there was no difference in the significance of the predictors of change in the group-level analysis. A lack of overlap meant that the difference was significant.

\section{Results}

The means, standard deviations, and correlations for the variables are shown in Table 1. In Table 2, the results of the fit indexes of the change score models are presented for each pair of mindfulness facets and burnout dimensions. All the models had a satisfactory fit with the data.

\section{Mindfulness Facets Mediated Intervention Effects on Burnout Dimensions}

Tables 3, 4, 5, 6, and 7 show the $\beta$ values and their confidence intervals for the models. The tables were grouped based on the mindfulness facets such that each table presented values for all three burnout dimensions when one of the mindfulness facets was considered. The description of the results was based on the values and significances of the coefficients, namely, $\beta_{2}, \beta_{3}, \beta_{9}$, $\beta_{10}, \beta_{2} \times \beta_{9}$, and $\beta_{2} \times \beta_{10}$. The intervention was effective in alleviating all burnout dimensions. All mindfulness facets improved during the intervention compared with the skills acquisition in the control group ( $\beta_{2}$ was significant with all combinations of mindfulness facets and burnout dimensions). There were both similarities and differences between the burnout dimensions in which mindfulness facets were mediators of change (based on the significances of the coefficients $\beta_{2}, \beta_{9}$, $\beta_{10}, \beta_{2} \times \beta_{9}$, and $\beta_{2} \times \beta_{10}$ and differences thereof).

Exhaustion Improvements in observing, acting with awareness, non-judging, and non-reacting during the intervention mediated the alleviation of exhaustion during the intervention (significant $\beta_{2}, \beta_{9}$, and $\beta_{2} \times \beta_{9}$ ). None of the significant four facets was more important than the others for the alleviation of exhaustion during the intervention (the confidence intervals overlapped between the facets). Only the improvement in non-judging during the intervention mediated the additional alleviation of exhaustion during the follow-up (significant $\beta_{2}$, $\beta_{10}$, and $\beta_{2} \times \beta_{10}$ ). The intervention had an additional direct effect on the alleviation of exhaustion, after controlling for the mediators (significant $\beta_{3}$ ).

Cynicism Improvements in all five mindfulness facets during the intervention mediated the alleviation of cynicism during the intervention. Improvements in describing and non-judging

Table 2 Model fits for change score models of mindfulness facets and burnout dimensions

\begin{tabular}{lllcccc}
\hline Mindfulness & Burnout & $\chi^{2}$ & $p$ value & RMSEA & CFI & TLI \\
\hline OB & EX & 215.78 & .0004 & 0.047 & 0.967 & 0.962 \\
DE & EX & 232.02 & $<.0001$ & 0.052 & 0.969 & 0.964 \\
AC & EX & 247.60 & $<.0001$ & 0.057 & 0.955 & 0.949 \\
NJ & EX & 182.47 & .0365 & 0.033 & 0.985 & 0.983 \\
NR & EX & 183.17 & .0338 & 0.033 & 0.982 & 0.980 \\
OB & CY & 194.58 & .0084 & 0.038 & 0.978 & 0.975 \\
DE & CY & 237.31 & $<.0001$ & 0.054 & 0.967 & 0.963 \\
AC & CY & 228.35 & $<.0001$ & 0.051 & 0.965 & 0.070 \\
NJ & CY & 170.23 & .1236 & 0.026 & 0.991 & 0.0650 \\
NR & CY & 155.74 & .3574 & 0.014 & 0.997 & 0.990 \\
OB & RE & 172.54 & .1004 & 0.027 & 0.987 & 0.997 \\
DE & RE & 222.71 & .0001 & 0.049 & 0.970 & 0.985 \\
AC & RE & 195.36 & .0075 & 0.039 & 0.977 & 0.966 \\
NJ & RE & 200.56 & .0037 & 0.041 & 0.975 & 0.973 \\
NR & RE & 142.20 & .6627 & 0.000 & 10.00 & 0.972 \\
\hline
\end{tabular}

$O B$ observing, $D E$ describing, $A C$ acting with awareness, $N J$ non-judging, $N R$ non-reacting, $E X$ exhaustion, $C Y$ cynicism, $R E$ reduced professional efficacy, $\chi^{2}$ chi-square test, RMSEA root mean square error of approximation, $C F I$ comparative fit index, TLI Tucker-Lewis index, SRMR standardized root mean square residual

A good model fit was assumed when CFI and TLI were close to 0.95 , the SRMR was close to 0.08 , and the RMSEA was close to 0.06 
Table $3 \beta$ values for the models of observing and three burnout dimensions

\begin{tabular}{|c|c|c|c|c|c|c|c|c|c|c|c|c|c|c|c|}
\hline & \multicolumn{5}{|c|}{ Exhaustion } & \multicolumn{5}{|l|}{ Cynicism } & \multicolumn{5}{|c|}{ Reduced professional efficacy } \\
\hline & Estimate & SE & $p$ & CI L & CI U & timate & SE & $p$ & I L & CI U & Estimate & SE & $p$ & CI L & CI U \\
\hline$\beta_{1}$ & 06 & 0.079 & .938 & 0.152 & 0.164 & 0.006 & 0.079 & .935 & -0.152 & 0.164 & - & 0.079 & .956 & -0.154 & 0.162 \\
\hline$\beta_{2}$ & 0.357 & 0.062 & $<.001$ & 0.233 & 0.481 & 0.358 & 0.062 & $<.001$ & 0.234 & 0.482 & 0.361 & 0.063 & $<.001$ & 0.235 & 0.487 \\
\hline$\beta_{3}$ & -0.205 & 0.073 & .005 & -0.351 & -0.059 & -0.126 & .076 & .098 & -0.278 & 0.026 & -0.126 & 0.086 & .142 & -0.298 & 0.046 \\
\hline$\beta_{4}$ & 0.083 & 0.078 & .289 & -0.073 & 0.239 & -0.066 & 77 & .390 & -0.220 & 0.088 & 0.021 & 0.085 & .803 & -0.149 & 0.191 \\
\hline$\beta_{5}$ & -0.466 & 0.081 & $<.001$ & -0.628 & -0.304 & -0.457 & 0 & $<.001$ & -0.617 & -0.297 & -0.443 & 0.083 & $<.001$ & -0.609 & -0.277 \\
\hline$\beta_{6}$ & -0.229 & 0.079 & .004 & -0.387 & -0.071 & -0.406 & 0.069 & $<.001$ & -0.544 & -0.268 & -0.074 & 0.094 & .430 & -0.262 & 0.114 \\
\hline$\beta_{7}$ & -0.237 & 0.092 & .010 & -0.421 & -0.053 & -0.249 & 0.088 & .005 & -0.425 & -0.073 & -0.252 & 0.088 & .004 & -0.428 & -0.076 \\
\hline$\beta_{8}$ & -0.203 & 0.117 & .083 & -0.437 & 0.031 & -0.312 & 0.127 & .014 & -0.566 & -0.058 & -0.366 & 0.133 & .006 & -0.632 & -0.100 \\
\hline$\beta_{9}$ & -0.324 & 0.108 & .003 & -0.540 & -0.108 & -0.225 & 0.080 & .005 & -0.385 & -0.065 & -0.176 & 0.104 & .092 & -0.384 & 0.032 \\
\hline$\beta_{10}$ & -0.133 & 0.087 & .128 & -0.307 & 0.041 & -0.147 & 0.075 & .049 & -0.297 & 0.003 & -0.293 & 0.078 & $<.001$ & -0.449 & -0.137 \\
\hline$\beta_{11}$ & -0.362 & 0.085 & $<.001$ & -0.532 & -0.192 & -0.304 & 0.082 & $<.001$ & -0.468 & -0.140 & -0.333 & 0.076 & $<.001$ & -0.485 & -0.181 \\
\hline$\beta_{2} \times \beta_{9}$ & -0.116 & 0.043 & .007 & -0.202 & -0.030 & -0.080 & 0.033 & 0.015 & -0.146 & -0.014 & -0.064 & 0.039 & .107 & -0.142 & 0.014 \\
\hline$\beta_{2} \times \beta_{10}$ & -0.047 & 0.034 & .162 & -0.115 & 0.021 & -0.053 & 0.028 & 0.061 & -0.109 & 0.003 & -0.106 & 0.035 & .002 & -0.176 & -0.036 \\
\hline
\end{tabular}

The most relevant $\beta$ values for answering the research questions are italicized

SE standard error, CI L 95\% confidence interval lower, CI U 95\% confidence interval upper

during the intervention mediated the additional alleviation of cynicism during the follow-up. None of the significant mindfulness facets (five during the intervention and two during the follow-up) was more important than the others for the alleviation of cynicism.

Reduced Professional Efficacy Improvements in describing, acting with awareness, non-judging, and non-reacting during the intervention mediated the alleviation of reduced professional efficacy during the intervention. Improvements in observing, describing, and non-judging during the intervention mediated the additional alleviation of reduced professional efficacy during the follow-up. None of the significant mindfulness facets (four during the intervention and three during the follow-up) was more important than the others for the alleviation of reduced professional efficacy.

Table $4 \beta$ values for the models of describing and three burnout dimensions

\begin{tabular}{|c|c|c|c|c|c|c|c|c|c|c|c|c|c|c|c|}
\hline & \multicolumn{5}{|c|}{ Exhaustion } & \multicolumn{5}{|l|}{ Cynicism } & \multicolumn{5}{|c|}{ Reduced professional efficacy } \\
\hline & Estimate & SE & $p$ & CI L & CI U & Estimate & SE & $p$ & CI L & CI U & Estimate & SE & $p$ & CI L & $\mathrm{CI} \mathrm{U}$ \\
\hline$\beta_{1}$ & 0.022 & 0.073 & .767 & -0.124 & 0.168 & 0.021 & 0.073 & .770 & -0.125 & 0.167 & 0.022 & 0.073 & .768 & -0.124 & 0.168 \\
\hline$\beta_{2}$ & 0.349 & 0.064 & $<.001$ & 0.221 & 0.477 & 0.348 & 0.064 & $<.001$ & 0.220 & 0.476 & 0.349 & 0.065 & $<.001$ & 0.219 & 0.479 \\
\hline$\beta_{3}$ & -0.246 & 0.073 & .001 & -0.392 & -0.100 & -0.117 & 0.071 & .099 & -0.259 & 0.025 & -0.115 & 0.086 & .179 & -0.287 & 0.057 \\
\hline$\beta_{4}$ & 0.083 & 0.078 & .285 & -0.073 & 0.239 & -0.066 & 0.077 & .388 & -0.220 & 0.088 & 0.021 & 0.086 & .809 & -0.151 & 0.193 \\
\hline$\beta_{5}$ & -0.407 & 0.070 & $<.001$ & -0.547 & -0.267 & -0.403 & 0.068 & $<.001$ & -0.539 & -0.267 & -0.396 & 0.071 & $<.001$ & -0.538 & -0.254 \\
\hline$\beta_{6}$ & -0.254 & 0.078 & .001 & -0.410 & -0.098 & -0.421 & 0.071 & $<.001$ & -0.563 & -0.279 & -0.056 & 0.097 & .564 & -0.250 & 0.138 \\
\hline$\beta_{7}$ & -0.146 & 0.084 & .084 & -0.314 & 0.022 & -0.156 & 0.082 & .058 & -0.320 & 0.008 & -0.160 & 0.083 & .055 & -0.326 & 0.006 \\
\hline$\beta_{8}$ & -0.255 & 0.117 & .029 & -0.489 & -0.021 & -0.328 & 0.132 & .013 & -0.592 & -0.064 & -0.342 & 0.132 & .010 & -0.606 & -0.078 \\
\hline$\beta_{9}$ & -0.173 & 0.097 & .072 & -0.367 & 0.021 & -0.250 & 0.094 & .008 & -0.438 & -0.062 & -0.217 & 0.101 & .032 & -0.419 & -0.015 \\
\hline$\beta_{10}$ & -0.185 & 0.097 & .056 & -0.379 & 0.009 & -0.165 & 0.077 & .032 & -0.319 & -0.011 & -0.230 & 0.082 & .005 & -0.394 & -0.066 \\
\hline$\beta_{11}$ & -0.311 & 0.074 & $<.001$ & -0.459 & -0.163 & -0.210 & 0.082 & .011 & -0.374 & -0.046 & -0.135 & 0.089 & .127 & -0.313 & 0.043 \\
\hline$\beta_{2} \times \beta_{9}$ & -0.060 & 0.036 & .096 & -0.132 & 0.012 & -0.087 & 0.037 & .019 & -0.161 & -0.013 & -0.076 & 0.037 & .042 & -0.150 & -0.002 \\
\hline$\beta_{2} \times \beta_{10}$ & -0.064 & 0.037 & .078 & -0.138 & 0.010 & -0.058 & 0.029 & .046 & -0.116 & 0.000 & -0.080 & 0.033 & .015 & -0.146 & -0.014 \\
\hline
\end{tabular}

The most relevant $\beta$ values for answering the research questions are italicized

$S E$ standard error, CI L 95\% confidence interval lower, CI U 95\% confidence interval upper 
Table $5 \quad \beta$ values for the models of acting with awareness and three burnout dimensions

\begin{tabular}{|c|c|c|c|c|c|c|c|c|c|c|c|c|c|c|c|}
\hline & \multicolumn{5}{|c|}{ Exhaustion } & \multicolumn{5}{|l|}{ Cynicism } & \multicolumn{5}{|c|}{ Reduced professional efficacy } \\
\hline & ite & SE & $p$ & I L & U & & SE & $p$ & $\mathrm{~L}$ & 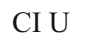 & & SE & $p$ & CI L & CI U \\
\hline$\beta_{1}$ & -0.108 & 0.071 & .127 & -0.250 & 0.034 & 0.110 & 0.072 & .126 & -0.254 & 0.034 & -0.110 & 0.071 & .124 & -0.252 & 0.03 \\
\hline$\beta_{2}$ & 0.181 & 0.056 & .001 & 0.069 & 0.293 & 0.186 & 0.057 & .001 & 0.072 & 0.300 & 0.184 & 0.057 & .001 & 0.070 & 0.298 \\
\hline$\beta_{3}$ & -0.238 & 0.070 & .001 & -0.378 & -0.098 & -0.130 & 0.073 & .073 & -0.276 & 0.016 & -0.100 & 0.083 & .227 & -0.266 & 0.066 \\
\hline$\beta_{4}$ & 0.083 & 0.078 & .287 & -0.073 & 0.239 & -0.069 & 0.077 & .372 & -0.223 & 0.085 & 0.025 & 0.086 & .771 & -0.147 & 0.197 \\
\hline$\beta_{5}$ & -0.581 & 0.053 & $<.001$ & -0.687 & -0.475 & -0.569 & 0.056 & $<.001$ & -0.681 & -0.457 & -0.571 & 0.055 & $<.001$ & -0.681 & -0.461 \\
\hline$\beta_{6}$ & -0.230 & 0.080 & .004 & -0.390 & -0.070 & 0.380 & 0.071 & $<.001$ & -0.522 & -0.238 & -0.015 & 0.090 & .866 & -0.195 & 0.165 \\
\hline$\beta_{7}$ & -0.265 & 0.094 & .005 & -0.453 & -0.077 & 0.266 & 0.092 & .004 & -0.450 & -0.082 & -0.268 & 0.089 & .003 & -0.446 & -0.09 \\
\hline$\beta_{8}$ & -0.233 & 0.113 & .040 & -0.459 & -0.007 & -0.221 & 0.130 & .088 & -0.481 & 0.039 & -0.273 & 0.119 & .022 & -0.511 & -0.035 \\
\hline$\beta_{9}$ & -0.293 & 0.082 & $<.001$ & -0.457 & -0.129 & -0.271 & 0.088 & .002 & -0.447 & -0.095 & -0.382 & 0.092 & $<.001$ & -0.566 & -0.198 \\
\hline$\beta_{10}$ & -0.114 & 0.087 & .190 & -0.288 & 0.060 & -0.139 & 0.074 & .061 & -0.287 & 0.009 & -0.193 & 0.077 & .012 & -0.347 & -0.039 \\
\hline$\beta_{11}$ & -0.414 & 0.081 & $<.001$ & -0.576 & -0.252 & -0.379 & 0.080 & $<.001$ & -0.539 & -0.219 & -0.293 & 0.085 & .001 & -0.463 & -0.12 \\
\hline$\beta_{2} \times \beta_{9}$ & -0.053 & 0.023 & .023 & -0.099 & -0.007 & -0.050 & 0.024 & .038 & -0.098 & -0.002 & -0.070 & 0.029 & .015 & -0.128 & -0.01 \\
\hline$\beta_{2} \times \beta_{10}$ & -0.021 & 0.017 & .224 & -0.055 & 0.013 & -0.026 & 0.016 & .099 & -0.058 & 0.006 & -0.036 & 0.018 & .053 & -0.072 & 0.00 \\
\hline
\end{tabular}

The most relevant $\beta$ values for answering the research questions are italicized

SE standard error, CI L 95\% confidence interval lower, CI U 95\% confidence interval upper

Summary of the Similarities and Differences Between Burnout Dimensions The improvements in the mindfulness facets mediated the intervention effects on all burnout dimensions both during the intervention and 10-month follow-up. Hence, the general hypothesis was supported. A large spread of mindfulness facets ( $4-5$ for each dimension) needed to improve to have significant reductions in burnout dimensions during the intervention. The differences in significant facets between the dimensions were minor when the short-term burnout change during the intervention was considered. However, there were some differences between the dimensions when the additional long-term burnout change during the 10-month follow-up was examined. Improvement in nonjudging mediated the changes in all burnout dimensions during the follow-up. For exhaustion, it was the only significant mediator during the follow-up, whereas for cynicism and

Table $6 \beta$ values for the models of non-judging and three burnout dimensions

\begin{tabular}{|c|c|c|c|c|c|c|c|c|c|c|c|c|c|c|c|}
\hline & \multicolumn{5}{|c|}{ Exhaustion } & \multicolumn{5}{|l|}{ Cynicism } & \multicolumn{5}{|c|}{ Reduced professional efficacy } \\
\hline & Estimate & SE & $p$ & CI L & CI U & Estimate & SE & $p$ & CI L & CI U & Estimate & SE & $p$ & CI L & $\mathrm{CI} \mathrm{U}$ \\
\hline$\beta_{1}$ & -0.136 & 0.075 & .069 & -0.286 & 0.014 & -0.137 & 0.076 & .069 & -0.289 & 0.015 & -0.140 & 0.075 & .062 & -0.290 & 0.010 \\
\hline$\beta_{2}$ & 0.238 & 0.069 & .001 & 0.100 & 0.376 & 0.240 & 0.068 & $<.001$ & 0.104 & 0.376 & 0.242 & 0.069 & $<.001$ & 0.104 & 0.380 \\
\hline$\beta_{3}$ & -0.215 & 0.070 & .002 & -0.355 & -0.075 & -0.086 & 0.070 & .221 & -0.226 & 0.054 & -0.090 & 0.084 & .288 & -0.258 & 0.078 \\
\hline$\beta_{4}$ & 0.083 & 0.078 & .285 & -0.073 & 0.239 & -0.068 & 0.077 & .375 & -0.222 & 0.086 & 0.030 & 0.088 & .736 & -0.146 & 0.206 \\
\hline$\beta_{5}$ & -0.382 & 0.062 & $<.001$ & -0.506 & -0.258 & -0.369 & 0.065 & $<.001$ & -0.499 & -0.239 & -0.376 & 0.063 & $<.001$ & -0.502 & -0.250 \\
\hline$\beta_{6}$ & -0.240 & 0.077 & .002 & -0.394 & -0.086 & -0.352 & 0.071 & $<.001$ & -0.494 & -0.210 & 0.032 & 0.101 & .754 & -0.170 & 0.234 \\
\hline$\beta_{7}$ & -0.311 & 0.080 & $<.001$ & -0.471 & -0.151 & -0.313 & 0.080 & $<.001$ & -0.473 & -0.153 & -0.307 & 0.081 & $<.001$ & -0.469 & -0.145 \\
\hline$\beta_{8}$ & -0.214 & 0.110 & .052 & -0.434 & 0.006 & -0.309 & 0.114 & .007 & -0.537 & -0.081 & -0.279 & 0.112 & .013 & -0.503 & -0.055 \\
\hline$\beta_{9}$ & -0.292 & 0.095 & .002 & -0.482 & -0.102 & -0.381 & 0.090 & $<.001$ & -0.561 & -0.201 & -0.363 & 0.100 & $<.001$ & -0.563 & -0.163 \\
\hline$\beta_{10}$ & -0.291 & 0.096 & .002 & -0.483 & -0.099 & -0.262 & 0.086 & .002 & -0.434 & -0.090 & -0.283 & 0.096 & .003 & -0.475 & -0.091 \\
\hline$\beta_{11}$ & -0.248 & 0.086 & .004 & -0.420 & -0.076 & -0.290 & 0.082 & $<.001$ & -0.454 & -0.126 & -0.306 & 0.105 & .004 & -0.516 & -0.096 \\
\hline$\beta_{2} \times \beta_{9}$ & -0.069 & 0.031 & .025 & -0.131 & -0.007 & -0.092 & 0.034 & .007 & -0.160 & -0.024 & -0.088 & 0.035 & .012 & -0.158 & -0.018 \\
\hline$\beta_{2} \times \beta_{10}$ & -0.069 & 0.028 & .012 & -0.125 & -0.013 & -0.063 & 0.029 & .030 & -0.121 & -0.005 & -0.068 & 0.031 & .029 & -0.130 & -0.006 \\
\hline
\end{tabular}

The most relevant $\beta$ values for answering the research questions are italicized

SE standard error, CI L 95\% confidence interval lower, CI U 95\% confidence interval upper 
Table $7 \quad \beta$ values for the models of non-reacting and three burnout dimensions

\begin{tabular}{|c|c|c|c|c|c|c|c|c|c|c|c|c|c|c|c|}
\hline & \multicolumn{5}{|c|}{ Exhaustion } & \multicolumn{5}{|l|}{ Cynicism } & \multicolumn{5}{|c|}{ Reduced professional efficacy } \\
\hline & te & SE & $p$ & CI L & CI U & & SE & $p$ & I L & $\mathrm{U}$ & nate & SE & $p$ & CI L & CI U \\
\hline$\beta_{1}$ & -0.124 & 0.074 & .093 & -0.272 & 0.024 & 0.125 & 0.074 & .092 & -0.273 & 0.023 & -0.126 & 0.074 & .088 & -0.274 & 0.02 \\
\hline$\beta_{2}$ & 0.273 & 0.069 & $<.001$ & 0.135 & 0.411 & 0.274 & 0.069 & $<.001$ & 0.136 & 0.412 & 0.274 & 0.069 & $<.001$ & 0.136 & 0.412 \\
\hline$\beta_{3}$ & -0.198 & 0.076 & .009 & -0.350 & -0.046 & 0.098 & 0.071 & .165 & -0.240 & 0.044 & -0.084 & 0.081 & .301 & -0.246 & 0.078 \\
\hline$\beta_{4}$ & 0.084 & 0.077 & .279 & -0.070 & 0.238 & 0.068 & 0.077 & .378 & -0.222 & 086 & 0.023 & 0.086 & .786 & -0.149 & 0.19 \\
\hline$\beta_{5}$ & 0.438 & 0.072 & $<.001$ & -0.582 & -0.294 & 0.428 & 070 & $<.001$ & -0.568 & -0.288 & -0.430 & 0.071 & $<.001$ & -0.572 & -0.28 \\
\hline$\beta_{6}$ & -0.246 & 0.078 & .002 & -0.402 & -0.090 & 0.368 & .071 & $<.001$ & -0.510 & -0.226 & -0.023 & 0.092 & .805 & -0.207 & 0.161 \\
\hline$\beta_{7}$ & -0.283 & 0.087 & .001 & -0.457 & -0.109 & -0.290 & .086 & .001 & -0.462 & -0.118 & -0.287 & 6 & .001 & -0.459 & -0.11 \\
\hline$\beta_{8}$ & -0.256 & 0.118 & .030 & -0.492 & -0.020 & -0.318 & 0.122 & .009 & -0.562 & -0.074 & -0.303 & 0.127 & .017 & -0.557 & -0.04 \\
\hline$\beta_{9}$ & -0.317 & 0.103 & .002 & -0.523 & -0.111 & -0.315 & 0.097 & .001 & -0.509 & -0.121 & -0.338 & 0.101 & .001 & -0.540 & -0.136 \\
\hline$\beta_{10}$ & -0.193 & 0.117 & .098 & -0.427 & 0.041 & -0.058 & 0.081 & .474 & -0.220 & 0.104 & -0.103 & 0.090 & .252 & -0.283 & 0.077 \\
\hline$\beta_{11}$ & -0.333 & 0.089 & <.001 & -0.511 & -0.155 & -0.344 & 0.073 & $<.001$ & -0.490 & -0.198 & -0.313 & 0.080 & $<.001$ & -0.473 & -0.153 \\
\hline$\beta_{2} \times \beta_{9}$ & -0.087 & 0.038 & .024 & -0.163 & -0.011 & -0.086 & 0.035 & .015 & -0.156 & -0.016 & -0.092 & 0.037 & .013 & -0.166 & -0.01 \\
\hline$\beta_{2} \times \beta_{10}$ & -0.053 & 0.034 & .125 & -0.121 & 0.015 & -0.016 & 0.023 & .490 & -0.062 & 0.030 & -0.028 & 0.027 & .295 & -0.082 & 0.02 \\
\hline
\end{tabular}

The most relevant $\beta$ values for answering the research questions are italicized

SE standard error, CI L 95\% confidence interval lower, CI U 95\% confidence interval upper

reduced professional efficacy, improvements in describing and observing were additionally needed. Based on confidence intervals, none of the significant facets appeared to be more important than others for the alleviation of burnout dimensions. However, improvement in non-judging systematically mediated both the short- and long-term reductions in all burnout dimensions and could thus be considered a central facet for burnout change. Exhaustion differed from the other burnout dimensions in a way that the intervention had an additional direct alleviating effect on it, after controlling for the mediators.

\section{Discussion}

This study investigated which of the mindfulness facets (observing, describing, acting with awareness, non-judging, and non-reacting) mediated the effects on different dimensions of burnout (exhaustion, cynicism, and reduced professional efficacy) during a MAV intervention and a 10-month follow-up. This study indicated that improving mindfulness skills using a MAV intervention could be effective in reducing burnout symptoms. Besides, the current MAV intervention appeared to be effective for all burnout dimensions, although, in previous studies, MAV interventions have consistently shown effects only on certain dimensions (Iancu et al. 2018). In the current study, mindfulness was an essential process behind the burnout reduction, as expected in theoretical models of interventions using mindfulness (Crane et al. 2017; Hayes et al. 2012). Furthermore, this study suggested that skills acquirement during an intervention can have long-lasting positive effects on burnout level even after the intervention, given that mindfulness improvement during the intervention mediated burnout alleviation also during the follow-up. The present findings were in line with previous mediation results of the role of mindfulness in burnout alleviation (Roeser et al. 2013). However, in this study, mediation effects were observed for an extended 10-month period compared with those of Roeser et al. (2013), which had a 3-month follow-up. This study also offered insights into the possible importance of the specific mindfulness facets for the alleviation of burnout symptoms.

\section{Similarities and Differences Between Burnout Dimensions in Mediators of Change}

The current study indicated that it could be essential to develop several mindfulness facets simultaneously to induce a significant change in burnout dimensions, although, in previous cross-sectional research, certain mindfulness facets have been more strongly associated with burnout (Kriakous et al. 2019; Taylor and Millear 2016). A large spread of the mindfulness facets mediated the change in burnout dimensions during the intervention, and the differences between the dimensions were minor. The importance of several mindfulness facets for shortterm burnout change can be understood in the context of burnout literature. Burnout is a persistent, job-related state of illbeing that has wide-ranging effects for the functioning of the employee (Leiter et al. 2014; Morse et al. 2012). Besides, Warr (2012) characterized burnout as a cognitive-affective disorder that comprises thoughts, feelings, and memories and encompasses a wide range of experiences in daily living. 
Because of the all-encompassing effects of burnout, likely, a profound change in a way a person views their situation is required to alleviate burnout. Hence, simultaneous improvement in several mindfulness facets could be needed to achieve such a change. Improvement in observing and describing skills could help a person identify the factors associated with burnout, whereas improvement in acting with awareness could help the person take action to affect these factors in a way that the possibility for recovery increases. Furthermore, better non-judging and non-reacting skills could help one adopt an accepting and defused stance towards even difficult experiences and focus their resources on recovery.

Some mindfulness facets even mediated the additional reduction of burnout during the 10-month follow-up. For a longterm change in burnout dimensions, the skills of non-judging (for all dimensions), describing (for cynicism and reduced professional efficacy), and observing (for reduced professional efficacy) appeared to be more relevant than the others. If several mindfulness facets are needed at the beginning of burnout recovery to induce profound change, the unique relevance of some mindfulness facets over the others could be more visible after a basic level of all skills is achieved. The significance of non-judging, describing, and observing for further alleviation of burnout can be understood in the context of the cognitive-affective description of burnout (Warr 2012). Exhaustion could be considered a more affective component of burnout since it refers to the feelings of emotional and physical strain after work (Näätänen et al. 2003). When a non-judgmental way to evaluate experiences increases, a person could experience more self-compassion and be less demanding when working. These changes could help to reduce the experienced strain and thus alleviate exhaustion. In turn, cynicism and professional efficacy could be described as more cognitive components of burnout, given that they involve interpretations of oneself, others, and situations (Näätänen et al. 2003). A new way to observe and describe experiences could be needed for one to continuously develop a non-judging stance towards their conceptualizations of themselves and others. On the other hand, non-judging skills could help one benefit from better observing and describing skills and change the interpretations of themselves and situations in a way that cynicism and reduced professional efficacy alleviate. The importance of joint development of observation-related facets (observing and describing) and reaction-related facets (nonjudging and non-reacting) have also been noticed with depression (Heeren et al. 2015; Querstret et al. 2018).

\section{Non-judging as a Central Skill in Burnout Change}

Only non-judging (measured here with reverse-scored items, e.g., "I tell myself I shouldn't be feeling the way I'm feeling." and "I make judgments about whether my thoughts are good or bad.") systematically mediated the changes in all burnout dimensions during both the intervention and the 10-month follow-up. Hence, improvement in non-judging skills could be the key to a long-term change in one's way of seeing themselves and adjusting their behavior to support burnout alleviation. There is often a discrepancy between the personal standards and perceived performance of people developing burnout symptoms (Ozbilir et al. 2015). Furthermore, low self-rated professional efficacy could lead to a situation where one refuses to rest and works even longer hours to compensate for one's perceived shortcomings. The improvement of nonjudging could help one process their expectations and develop a less guilt-inducing attitude towards themselves. When nonjudging skills are improved, it could be easier to recognize the over-demanding expectations and change one's behavior accordingly. Better non-judging skills could also help to adopt a more self-compassionate way to act with these expectations. One could acknowledge the feelings of exhaustion and give space for recovery in daily living. Non-judging stance towards experiences could also prevent automatic negative evaluations of job conditions and thereby alleviate cynicism. In line with the present findings, high non-judging has been linked to lower levels of exhaustion and cynicism in cross-sectional research (Taylor and Millear 2016).

Interestingly, in the current study, the role of acting with awareness was not as pronounced as in cross-sectional burnout studies (Kriakous et al. 2019; Yang et al. 2017). Compared with the present sample that included only those with high initial burnout, the participants in the previous studies experienced varying levels of burnout symptoms (Kriakous et al. 2019; Yang et al. 2017). Acting with awareness could be an important skill to mitigate the risk factors for developing severe burnout. Furthermore, when burnout symptoms are mild, there could be more resources for acting with conscious intention to improve one's well-being. However, when exhaustion has depleted resources of the employee, and when the evaluations of one's capabilities to handle job demands have become more negative, taking conscious action to change the situation could be overwhelming. Thus, when severe burnout has developed, improving non-judging skills could be more beneficial in helping the person give time for rest and observe themselves and the situation with less judgment and negativity. The current findings indicated that with a MAV intervention, non-judging skills could be improved to a level that yields positive long-term effects on burnout. This result is encouraging for the further study of cost-effective ways to increase relevant skills for burnout alleviation.

\section{Limitations and Future Research}

More research is needed to generate conclusions on the associations between mindfulness facets and burnout dimensions. In this study, mindfulness and burnout were investigated with self-report measures, which raised the possibility of common 
method bias. Nevertheless, these phenomena are best evaluated by using self-reports, while the use of longitudinal data diminishes the likelihood of common method bias (Doty and Glick 1998). The differences between burnout dimensions regarding essential mindfulness facets were evaluated based on confidence intervals, which showed maximums and minimums for the mediation effects on the present sample. With a larger sample size, the non-significant mediators could become significant. Before the post-measurement, people experiencing more exhaustion and cynicism were more likely to drop out, which raised the question of whether the intervention asked too much of the participants. However, all the participants had high initial burnout, as evaluated by the BBI (Näätänen et al. 2003). Most completed the intervention, and the dropout rate during the follow-up was comparable to that of Roeser et al. (2013) with shorter follow-up. The generalizability of the results is restricted because the sample mainly comprised highly educated women. This group could have been more motivated to participate in an intervention that contained a substantial amount of homework and group participation. However, the investigated intervention was not an exception in the requirement of homework and participation (Luken and Sammons 2016). More research is needed on whether interventions similar to that investigated in the current study are viable, for example, for less educated or male working populations. Further study is needed to determine whether the present mediation results could be replicated in other interventions using mindfulness. Furthermore, other processes affecting MAV intervention results should be studied besides mindfulness processes since the present intervention had an additional direct effect on exhaustion, after controlling for the mediators.

Author Contributions SMK: designed and executed the study, assisted in the data analyses, and wrote the paper. AP: led the research project and planning of the intervention and aided in the study design and paper writing. AT: analyzed the data and wrote part of the "Method" and "Results" sections. AM: collaborated in the study design and paper writing. RL: collaborated in the study design and paper writing. All authors approved the final version of the manuscript for submission.

Funding Information Open access funding provided by University of Jyväskylä (JYU).

Data Availability All data related to this study are available from the authors upon request. The data are not yet publicly available because the project group is still processing it.

\section{Compliance with Ethical Standards}

Conflict of Interest The authors declare that they have no conflict of interest.

Ethics Statement This study was conducted in compliance with the ethical standards of APA and the institutional and national research committee, as well as following the 1964 Declaration of Helsinki, its later amendments, and comparable ethical standards. Ethical approval was provided by the Research Ethics Committee of the Central Finland Health Care District.

Informed Consent Statement All participants provided informed consent.

Open Access This article is licensed under a Creative Commons Attribution 4.0 International License, which permits use, sharing, adaptation, distribution and reproduction in any medium or format, as long as you give appropriate credit to the original author(s) and the source, provide a link to the Creative Commons licence, and indicate if changes were made. The images or other third party material in this article are included in the article's Creative Commons licence, unless indicated otherwise in a credit line to the material. If material is not included in the article's Creative Commons licence and your intended use is not permitted by statutory regulation or exceeds the permitted use, you will need to obtain permission directly from the copyright holder. To view a copy of this licence, visit http://creativecommons.org/licenses/by/4.0/.

\section{References}

Ahola, K., Toppinen-Tanner, S., \& Seppänen, J. (2017). Interventions to alleviate burnout symptoms and to support return to work among employees with burnout: systematic review and meta-analysis. Burnout Research, 4, 1-11. https://doi.org/10.1016/j.burn.2017.02. 001.

Baer, R. A., Smith, G. T., Hopkins, J., Krietemeyer, J., \& Toney, L. (2006). Using self-report assessment methods to explore facets of mindfulness. Assessment, 13(1), 27-45. https://doi.org/10.1177/ 1073191105283504.

Baer, R. A., Smith, G. T., Lykins, E., Button, D., Krietemeyer, J., Sauer, S., Walsh, E., Duggan, D., \& Williams, J. M. G. (2008). Construct validity of the Five Facet Mindfulness Questionnaire in meditating and nonmeditating samples. Assessment, 15(3), 329-342. https:// doi.org/10.1177/1073191107313003.

Bergman, A. L., Christopher, M. S., \& Bowen, S. (2016). Changes in facets of mindfulness predict stress and anger outcomes for police officers. Mindfulness, 7(4), 851-858. https://doi.org/10.1007/ s12671-016-0522-z.

Crane, R. S., Brewer, J., Feldman, C., Kabat-Zinn, J., Santorelli, S., Williams, J. M. G., \& Kuyken, W. (2017). What defines mindfulness-based programs? The warp and the weft. Psychological Medicine, 47(6), 990-999. https://doi.org/10.1017/ S0033291716003317.

Doty, D. H., \& Glick, W. H. (1998). Common methods bias: does common methods variance really bias results? Organizational Research Methods, 1(4), 374-406. https://doi.org/10.1177/ 109442819814002.

Fletcher, L., \& Hayes, S. C. (2005). Relational frame theory, acceptance and commitment therapy, and a functional analytic definition of mindfulness. Journal of Rational-Emotive \& Cognitive-Behavior Therapy, 23(4), 315-336. https://doi.org/10.1007/s10942-005$0017-7$.

Hayes, S. C., \& Hofmann, S. G. (2017). The third wave of cognitive behavioral therapy and the rise of process-based care. World Psychiatry, 16(3), 245-246. https://doi.org/10.1002/wps.20442.

Hayes, S. C., Pistorello, J., \& Levin, M. E. (2012). Acceptance and commitment therapy as a unified model of behavior change. The Counseling Psychologist, 40, 976-1002. https://doi.org/10.1177/ 0011000012460836. 
Heeren, A., Deplus, S., Peschard, V., Nef, F., Kotsou, I., Dierickx, C., Mondillon, L., Robinaugh, D. J., \& Philippot, P. (2015). Does change in self-reported mindfulness mediate the clinical benefits of mindfulness training? A controlled study using the French translation of the Five Facet Mindfulness Questionnaire. Mindfulness, 6(3), 553-559. https://doi.org/10.1007/s12671-014-0287-1.

Hofmann, S. G., \& Hayes, S. C. (2019). The future of intervention science: process-based therapy. Clinical Psychological Science: A Journal of the Association for Psychological Science, 7(1), 37-50. https://doi.org/10.1177/2167702618772296.

Hu, L., \& Bentler, P. M. (1999). Cutoff criteria for fit indexes in covariance structure analysis: conventional criteria versus new alternatives. Structural Equation Modeling, 6(1), 1-55. https://doi.org/10. 1080/10705519909540118.

Iancu, A. E., Rusu, A., Măroiu, C., Păcurar, R., \& Maricuțoiu, L. P. (2018). The effectiveness of interventions aimed at reducing teacher burnout: a meta-analysis. Educational Psychology Review, 30(2), 373-396. https://doi.org/10.1007/s10648-017-9420-8.

Kriakous, S. A., Elliott, K. A., \& Owen, R. (2019). Coping, mindfulness, stress, and burnout among forensic health care professionals. Journal of Forensic Psychology Research and Practice, 19(2), 128-146. https://doi.org/10.1080/24732850.2018.1556545.

Leiter, M. P., Bakker, A. B., \& Maslach, C. (2014). Burnout at work: a psychological perspective. East Sussex: Psychology Press.

Little, T. D., Cunningham, W. A., Shahar, G., \& Widaman, K. F. (2002). To parcel or not to parcel: exploring the question, weighing the merits. Structural Equation Modeling, 9(2), 151-173. https://doi. org/10.1207/S15328007SEM0902_1.

Lloyd, J., Bond, F. W., \& Flaxman, P. E. (2013). The value of psychological flexibility: examining psychological mechanisms underpinning a cognitive behavioural therapy intervention for burnout. Work and Stress, 27(2), 181-199. https://doi.org/10.1080/02678373. 2013.782157.

Luken, M., \& Sammons, A. (2016). Systematic review of mindfulness practice for reducing job burnout. The American Journal of Occupational Therapy, 70, 1-13. https://doi.org/10.5014/ajot. 2016.016956

Mäkikangas, A., \& Kinnunen, U. (2016). The person-oriented approach to burnout: a systematic review. Burnout Research, 3, 11-23. https://doi.org/10.1016/j.burn.2015.12.002.

McArdle, J. J. (2009). Latent variable modeling of differences and changes with longitudinal data. Annual Review of Psychology, 60, 577605. https://doi.org/10.1146/60.110707.163612.

McArdle, J. J., \& Hamagami, F. (2001). Latent difference score structural models for linear dynamic analyses with incomplete longitudinal data. In L. M. Collins \& A. G. Sayer (Eds.), Decade of behavior. New methods for the analysis of change (pp. 139-175). Washington, DC: American Psychological Association. https://doi.org/10.1037/ 10409-005.

Morse, G., Salyers, M. P., Rollins, A. L., Monroe-DeVita, M., \& Pfahler, C. (2012). Burnout in mental health services: a review of the problem and its remediation. Administration and Policy in Mental Health and Mental Health Services Research, 39(5), 341-352. https://doi. org/10.1007/s10488-011-0352-1.

Muthén, L. K., \& Muthén, B. O. (2017). Mplus user's guide (8th ed.). Los Angeles: Muthén \& Muthén.

Näätänen, P., Aro, A., Matthiesen, S., \& Salmela-Aro, K. (2003). Bergen burnout indicator 15. Helsinki: Edita.
Ozbilir, T., Day, A., \& Catano, V. M. (2015). Perfectionism at work: an investigation of adaptive and maladaptive perfectionism in the workplace among Canadian and Turkish employees. Applied Psychology, 64(1), 252-280. https://doi.org/10.1111/apps.12032.

Puolakanaho, A., Tolvanen, A., Kinnunen, S. M., \& Lappalainen, R. (2020). A psychological flexibility -based intervention for burnout: a randomized controlled trial. Journal of Contextual Behavioral Science, 15, 52-67. https://doi.org/10.1016/j.jcbs.2019.11.007.

Querstret, D., Cropley, M., \& Fife-Schaw, C. (2016). Internet-based instructor-led mindfulness for work-related rumination, fatigue, and sleep: assessing facets of mindfulness as mechanisms of change. A randomized waitlist control trial. Journal of Occupational Health Psychology, 22(2), 153-169. https://doi.org/10.1037/ocp0000028.

Querstret, D., Cropley, M., \& Fife-Schaw, C. (2018). The effects of an online mindfulness intervention on perceived stress, depression and anxiety in a non-clinical sample: a randomised waitlist control trial. Mindfulness, 9(6), 1825-1836. https://doi.org/10.1007/s12671-0180925-0.

Roeser, R. W., Schonert-Reichl, K. A., Jha, A., Cullen, M., Wallace, L., Wilensky, R., Oberle, E., Thomson, K., Taylor, C., \& Harrison, J. (2013). Mindfulness training and reductions in teacher stress and burnout: results from two randomized, waitlist-control field trials. Journal of Education \& Psychology, 105(3), 787-804. https://doi. org/10.1037/a0032093.

Salmela-Aro, K., Rantanen, J., Hyvönen, K., Tilleman, K., \& Feldt, T. (2011). Bergen Burnout Inventory: reliability and validity among Finnish and Estonian managers. International Archives of Occupational and Environmental Health, 84(6), 635-645. https:// doi.org/10.1007/s00420-010-0594-3.

Taylor, N. Z., \& Millear, P. M. R. (2016). The contribution of mindfulness to predicting burnout in the workplace. Personality and Individual Differences, 89, 123-128. https://doi.org/10.1016/j.paid. 2015.10.005.

Warr, P. (2012). How to think about and measure psychological wellbeing. In R. R. Sinclair, M. Wang, \& L. E. Tetrick (Eds.), Research methods in occupational health psychology: measurement, design, and data analysis (pp. 76-90). Abingdon: Routledge.

Waters, C. S., Frude, N., Flaxman, P. E., \& Boyd, J. (2018). Acceptance and commitment therapy (ACT) for clinically distressed health care workers: waitlist-controlled evaluation of an ACT workshop in a routine practice setting. The British Journal of Clinical Psychology, 57(1), 82-98. https://doi.org/10.1111/bjc.12155.

Williams, M., \& Penman, D. (2011). Mindfulness: a practical guide to finding peace in a frantic world. London: Piatkus.

Wolf, E. J., Harrington, K. M., Clark, S. L., \& Miller, M. W. (2013). Sample size requirements for structural equation models: an evaluation of power, bias, and solution propriety. Educational and Psychological Measurement, 73(6), 913-934. https://doi.org/10. 1177/0013164413495237.

Yang, S., Meredith, P., \& Khan, A. (2017). Is mindfulness associated with stress and burnout among mental health professionals in Singapore? Psychology, Health \& Medicine, 22(6), 673-679. https://doi.org/10.1080/13548506.2016.1220595.

Publisher's Note Springer Nature remains neutral with regard to jurisdictional claims in published maps and institutional affiliations. 